\section{CHARITY ACCREDITS BAMBOO TOOTHBRUSH}

The Oral Health Foundation has given its seal of approval to the Bambooth series of toothbrushes, which help aid good oral health and provide an environmentally friendly alternative to plastic brushes.

The toothbrush handles are made from muso bamboo, the world's fastest-growing renewable resource, which is also completely biodegradable. The brush also has a thinner neck for better handling in the mouth.

Dr Ben Atkins, President of the Oral

Health Foundation, believes that this marks a great moment for the charity's accreditation programme: 'Most of us are looking for more ways to be kinder to the environment by ditching plastic and moving to natural and sustainable alternatives. This has traditionally been very difficult in oral care as single use plastics are extremely common. That's why it is a terrific feeling to be able to accredit our first bamboo toothbrush.

The Oral Health Foundation's accreditation programme examines product claims to make sure they are reliable and can be supported by scientific evidence. Each product that passes through the charity's expert panel is awarded with a kite mark to show that its claims have been rigorously checked and effectively verified.
Co-Founder of Bambooth, Niamh McGill, is delighted by the Oral Health Foundation's accreditation and explained why more people should consider making the switch to environmentally friendly brushes: 'More than 3.5 billion plastic toothbrushes are made every year and many of these end up in oceans and landfill. This causes pollution, harms wildlife and affects the food chain. By moving to a bamboo toothbrush, you and your patients can make a big difference.'

To find out more about Bambooth visit www.bambooth.com.

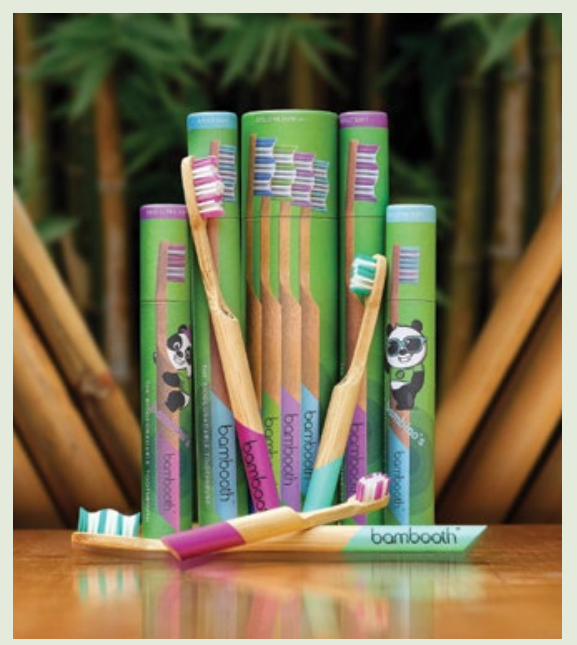

DON'T LET PPE WASTE BECOME A NEW

\section{PROBLEM}

With increased demand for singleuse PPE comes an increased amount of waste. Disposing of this waste responsibly is essential for ensuring the safety of others and the environment.

Initial Medical are experts in healthcare waste management and offer a full range of products, services and support to help dental practices optimise their waste management.

Initial Medical can help you create a dedicated PPE disposal area in your waiting room with their specifically designed 60L capacity bags and SilverSafe, pedal-operated clinical bins. They are colour-coded orange to meet all legal recommendations and best practice protocols for the safe, effective and ethical disposal of potentially infection waste. These are in addition to your normal clinical waste containers in the surgeries.

Initial Medical also offer a secure wheelie bin for lockable and fullycompliant storage, which it will collect at suitable intervals and ensure its safe disposal.

Ensure the appropriate disposal of PPE waste throughout your practice to stop it from becoming a new problem.

For further information visit www. initial.co.uk/medical or tel: 0870850 4045 .

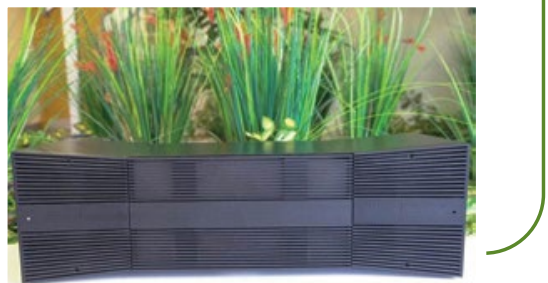

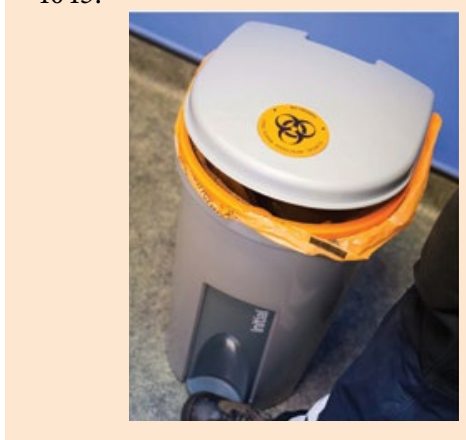

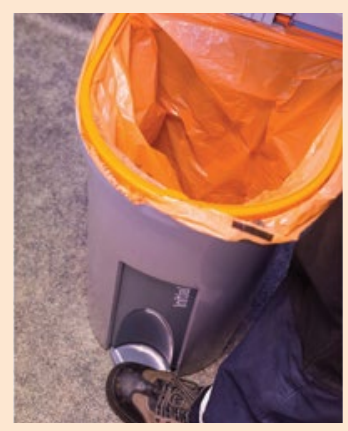

The PFAU machines are also recognised to improve asthma, hay fever and other respiratory conditions and can even reduce snoring, alongside, removing odours such as smoke, pet, and damp.

For more information call 01253736355 or email account@dentaldecontamination.co.uk.

filtration systems, the PFAU was also found to be the only one which, as well as removing unpleasant odours and particles from the air, actually destroyed air-borne bacteria and microorganisms.

PFAUs have been found to be 360 times more effective than standard air purifiers.

The PFAUs can achieve reduction in fallow time by totally changing the air a minimum of it sterilises the air to an unprecedented $\log 10$ status. It kills all airborne bacteria and destroys viruses, including the common cold, human flu, MRSA and enveloped viruses.

These systems will give both clients and employees the total peace of mind that their workplace is a safe and sterile environment.

Laboratory tests show that whereas the market leading conventional air purifier took two-and-half days to clean the air in a room, PFAUs took seven-and-half minutes. To the delight of the testing team, as well as being hugely more effective than conventional air 